\title{
Circulating VEGF as a biological marker in patients with rheumatoid arthritis? Preanalytical and biological variability in healthy persons and in patients
}

\author{
Merete Lund Hetland ${ }^{\mathrm{a}, *}$, Ib Jarle Christensen ${ }^{\mathrm{b}}$, Tine Lottenburger ${ }^{\mathrm{c}}$, Julia Sidenius Johansen ${ }^{\mathrm{d}}$, \\ Mads Nordahl Svendsen ${ }^{\mathrm{b}}$, Kim Hørslev-Petersen ${ }^{\mathrm{c}}$, Lone Nielsen ${ }^{\mathrm{e}}$ and Hans Jørgen Nielsen ${ }^{\mathrm{b}}$ \\ ${ }^{a}$ Department of Rheumatology, Copenhagen University Hospital, Hvidovre, Denmark \\ ${ }^{\mathrm{b}}$ Department of Surgical Gastroenterology, Copenhagen University Hospital, Hvidovre, Denmark \\ ${ }^{\mathrm{c}}$ Rheumatism Hospital, University of Southern Denmark, Gråsten, Denmark \\ ${ }^{\mathrm{d}}$ Department of Rheumatology, Copenhagen University Hospital, Herlev, Denmark \\ e Department of Clinical Immunology, Nykфbing Falster Hospital, Denmark
}

\begin{abstract}
Background: Soluble vascular endothelial growth factor (VEGF) is a promising biomarker in monitoring rheumatoid arthritis (RA), but studies of pre-analytical and biologic variability are few.

Methods: VEGF was measured by ELISA methods in serum and plasma from healthy persons and RA patients. Pre-analytical factors were investigated. A reference interval for VEGF was established in serum and plasma from 306 healthy persons. Diurnal, day-to-day, week-to-week, long-term variability, and impact of exercise were evaluated.

Results: Delayed processing time, room temperature, low centrifugal force and contamination of plasma with cellular elements lead to significant increases in VEGF levels, whereas storage for up to 2 years at $-80^{\circ} \mathrm{C}$ or up to 10 freeze/thaw cycles did not affect VEGF levels. Serum VEGF levels were 7-10 fold higher than plasma VEGF levels. Reference intervals for VEGF (plasma: $45 \mathrm{pg} / \mathrm{ml}$ (range: non-detectable to 352); serum: $328 \mathrm{pg} / \mathrm{ml}$ (53-1791)) were independent of gender and age. Shortand long-term biologic variability included diurnal variation (sampling should take place after 7 AM) and impact of exercise (increased VEGF immediately after bicycling normalised within 1 hour).

Conclusions: Pre-analytical factors and biologic variability including diurnal variation and impact of exercise should be accounted for in future studies that include circulating VEGF as a biological marker.
\end{abstract}

Keywords: Rheumatoid arthritis, vascular endothelial growth factor, exercise, biological marker

\section{Introduction}

Rheumatoid arthritis (RA) is an inflammatory disease of unknown aetiology. Angiogenesis appears to

\footnotetext{
*Corresponding author: Merete Lund Hetland, MD, PHD, Department of Rheumatology 232, Copenhagen University Hospital at Hvidovre, Kettegaard Alle 30, DK-2650 Hvidovre, Denmark. Tel.: +45 36322277; Fax: +45 36471410; E-mail: merete. hetland@dadlnet.dk.
}

be an important factor in the pathogenesis of RA [6, $12,19,25]$. The inflamed synovium in active RA is histologically characterized by angiogenesis with loss of the normal, vascular structure. The protein vascular endothelial growth factor (VEGF) plays a critical role in angiogenesis. It stimulates proliferation and migration of endothelial cells to form new blood vessels and increases the permeability of the vessels $[1,5,11,20]$. There is increased expression of VEGF in macrophages and fibroblasts in the synovial membrane from RA pa- 
tients $[6,13,21]$ and VEGF is present in the synovial fluid of RA patients $[6,9,13,18]$.

The serum VEGF concentration has been reported to be elevated in patients with active RA compared to healthy persons and to be correlated to serum C-reactive protein, the number of swollen joints and disease activity score $[2,9,16,18,24]$. Serum VEGF is decreased in RA patients, who respond to medical treatment [2,9, 18 , and in vitro studies have shown decreased VEGF synthesis in synovial cells from RA patients after stimulation with steroid [18]. In one study serum VEGF levels in patients with early RA was predictive of progression of joint destruction after one year [2].

Based on these observations, serum VEGF has been suggested as a potential biomarker in monitoring of disease activity, treatment response, erosive disease and extra-articular involvement in RA patients $[2,9,15,17$, 22]. Circulating biomarkers are, however, subject to variability arising from sampling procedures and biologic variation, and this variability must be determined and adjusted for in the interpretation of laboratory results.

The aims of the present study were to determine the methodological variability, short- and long-term biological variability in healthy persons and RA patients, to establish a reference interval in healthy persons, and to investigate differences in serum and plasma concentrations of VEGF.

\section{Materials and methods}

\subsection{VEGF ELISA}

Serum and plasma concentrations of VEGF were determined by a commercially available human VEGF quantitative enzyme-linked immunosorbent assay (ELISA) (R\&D Systems, Abingdon, Oxon, UK) in accordance with the manufacturer's instructions. The monoclonal antibodies in the ELISA are raised in mice against recombinant human $\mathrm{VEGF}_{165}$.

$100 \mu \mathrm{l}$ serum or plasma was used for each analysis. All samples and standards were analysed in duplicate, and the mean value was used for statistical calculations. All samples from one subject were analyzed on one ELISA plate. Reanalysis was done if the coefficient of variation $(\mathrm{CV})$ of the mean value was $>10 \%$. Samples with values higher than the highest standard (2000 pg/ml) were diluted. Optical densities were determined using a microplate reader (Spectra II, Wallac, Austria) at $450 \mathrm{~nm}$. A standard curve was estimated using a smoothed spline function. The calibration on each microplate used recombinant human VEGF standards and three internal standards from our laboratory.

\subsection{Methodological variability}

\subsubsection{Pre-analytical handling}

Serum and EDTA plasma samples were obtained at room temperature. The samples were centrifuged after 30 minutes at $1700 \mathrm{~g}$ for 10 minutes at $4{ }^{\circ} \mathrm{C}$ and stored at $-80^{\circ} \mathrm{C}$ until analysis unless otherwise stated below.

\subsubsection{Detection limit}

The detection limit was assessed 1) by calculating the limit of the blank (a signal 3SD above the mean for a calibrator that is free of analyte) $(n=20) ; 2)$ by the $15.6 \mathrm{pg} / \mathrm{ml}$ standard $(n=20)$; and 3 ) by a pooled patient sample with a plasma VEGF level of $16 \mathrm{pg} / \mathrm{ml}$ $(n=20)$.

\subsubsection{Intra- and interassay $C V$}

Three standards from two patient plasma pools were included on each plate: Mean VEGF concentration $374 \mathrm{pg} / \mathrm{ml} ; 487 \mathrm{pg} / \mathrm{ml}$ and $216 \mathrm{pg} / \mathrm{ml}$ (diluted 1:2).

\subsubsection{Recovery}

VEGF standard (2000 pg/ml) (dilution 1:2, 1:4 and $1: 8)$ was added to three plasma pools $(30,311$, and 425 $\mathrm{pg} / \mathrm{ml}$ ) and corresponding serum pools $(346,913$, and $1050 \mathrm{pg} / \mathrm{ml}$ ). Original and added pools were measured 3 times in duplicate.

\subsubsection{Delay and temperature before centrifugation}

Corresponding serum and plasma samples were collected from 10 healthy persons and 5 RA patients. From each individual, 8 serum and 8 EDTA plasma tubes were kept at room temperature, and a similar number was kept at $4^{\circ} \mathrm{C}$. After 30, 45, $60 \mathrm{~min}, 2,3,4,6$ and 24 hours, one serum and one EDTA tube were centrifuged at $2000 \mathrm{~g}$ for 10 minutes at room temperature and the serum and plasma were stored at $-80^{\circ} \mathrm{C}$ until analysis.

\subsubsection{Centrifugation and strata}

Corresponding samples of plasma and serum from 12 healthy persons and 12 RA patients were centrifuged at different centrifugal forces $(\mathrm{g})$. From each tube the upper, medium, lower and the lowest $0.5 \mathrm{~cm}$ of the supernatant was aliquoted into separate tubes.

\subsubsection{Buffy coat contamination}

In 18 healthy persons, plasma was collected on ice and centrifuged at $2500 \mathrm{~g}$. The supernatant (except the $5 \mathrm{~mm}$ above the buffy coat) was mixed and aliquoted into 4 tubes. The remaining $5 \mathrm{~mm}$ of the supernatant was added to tube 1 . Contamination with cellular elements could be disclosed in tube 1 compared with the 3 non-contaminated tubes. 


\subsubsection{Different types of blood samples and temperature}

Corresponding samples of serum, heparin plasma, citrate plasma, EDTA plasma (all stored at room temperature, the latter also stored on ice for 30 minutes,) were collected from 10 healthy persons and 10 RA patients. After centrifugation the serum and plasma were aliquoted (except the $5 \mathrm{~mm}$ stratum above the buffy coat). VEGF concentrations in citrate plasma were corrected for buffer volume.

\subsubsection{Repeated thawing}

Corresponding serum and EDTA plasma samples were collected from 10 healthy persons and 10 RA patients. The serum and EDTA plasma samples were stored at $-80^{\circ} \mathrm{C}$ and thawed/refrozen from 0 to 10 times before analysis.

\subsubsection{Storage}

Plasma VEGF in 80 RA patients was measured shortly after blood collection. 2 years later, the samples were thawed and reanalysed.

\subsection{Biological variability}

\subsubsection{Preanalytical handling}

Serum and EDTA plasma samples were obtained at room temperature, centrifuged within 2 hours at 2500 $\mathrm{g}$ for 10 minutes at $4{ }^{\circ} \mathrm{C}$ and stored at $-80^{\circ} \mathrm{C}$ until analysis, unless otherwise stated below.

\subsubsection{Reference interval (normal range)}

VEGF was determined in serum and plasma from 306 healthy, volunteer blood donors, who were well characterized: A health questionnaire was filled out and approved before every blood donation, and donation was not allowed during disease periods.

\subsubsection{Short-term (diurnal) variability}

Diurnal variability was determined in three populations: 1) Early, untreated RA ( $n=11)$; 2) Long-term erosive RA $(n=10) ; 3)$ Healthy persons $(n=16)$. All study subjects were hospitalized and had uniform schedules for meals and sleep. They were not permitted to exercise. Plasma was collected at seven time points during a $24 \mathrm{~h}$ period (10 AM, $1 \mathrm{PM}, 4 \mathrm{PM}, 7 \mathrm{PM}, 10$ $\mathrm{PM}, 7 \mathrm{AM}$ and $10 \mathrm{AM}$ ) and centrifuged at $1200 \mathrm{~g}$.

\subsubsection{Variability during 3 weeks}

VEGF was determined in plasma samples collected at $8 \mathrm{AM}$ on day $1,2,8,15,22$ in thirty-two persons recruited among hospital staff. In addition, VEGF was determined in plasma collected at $2 \mathrm{PM}$ at day 8 .

\subsubsection{Long-term variability}

The 3 weeks variability study was repeated after 6 $\left(2^{\text {nd }}\right.$ round) and 12 months ( $3^{\text {rd }}$ round).

\subsubsection{Exercise}

Plasma VEGF was collected before physical exercise, immediately after exercise (25 minutes bicycling with incremental work load every 4 minutes), and again 1 and 3 hours post-exercise in: 1) Patients with early, untreated RA ( $n=10)$; 2) Patients with long-term erosive RA $(n=10)$; and 3$)$ Healthy persons $(n=14)$.

\subsection{Ethics}

All participants gave their written informed consent. The local ethics committees approved the studies, which were carried out in accordance with the Declaration of Helsinki.

\subsection{Statistical analyses}

Descriptive statistics for VEGF levels are presented by the median or the geometric mean, and range. The variation over time is given by the CV. The CV's of VEGF levels analyzed over time (variability over 3 weeks and long-term variability studies) were compared to the intra-assay CV. Serum and plasma samples of VEGF were compared using Wilcoxon's signed rank test and Spearman's rank correlation. Association of VEGF to gender and age were done using Wilcoxon's rank sum test and Spearman's rank correlation. The relation between VEGF levels and time to processing at either $4^{\circ} \mathrm{C}$ or room temperature was analysed using linear regression with VEGF level and time log transformed. VEGF levels in the analysis of circadian variation and physical activity were studied using a general linear model with repeated measures. Estimates were obtained by generalized estimating equations assuming a normal distribution on the log scale. P-values less than 5\% were considered significant. All statistical calculations were done using SAS (Statistical analysis system, version 9.1, SAS Institute, Cary, NC, USA).

\section{Results}

\subsection{Metodological variability}

\subsubsection{Detection limit}

The limit of the blank was $11.6 \mathrm{pg} / \mathrm{ml}$. The detection limit based on the $15.6 \mathrm{pg} / \mathrm{ml}$ standard was mean 10.9 $\mathrm{pg} / \mathrm{ml}$ (CV: $14.4 \%)$, and based on the patient sample mean $12.7 \mathrm{pg} / \mathrm{ml}$ (CV: 20.6\%). These corresponded satisfactory with the manufacturer's specifications of a sensitivity of $9 \mathrm{pg} / \mathrm{ml}$ and a detection limit of $16 \mathrm{pg} / \mathrm{ml}$. 

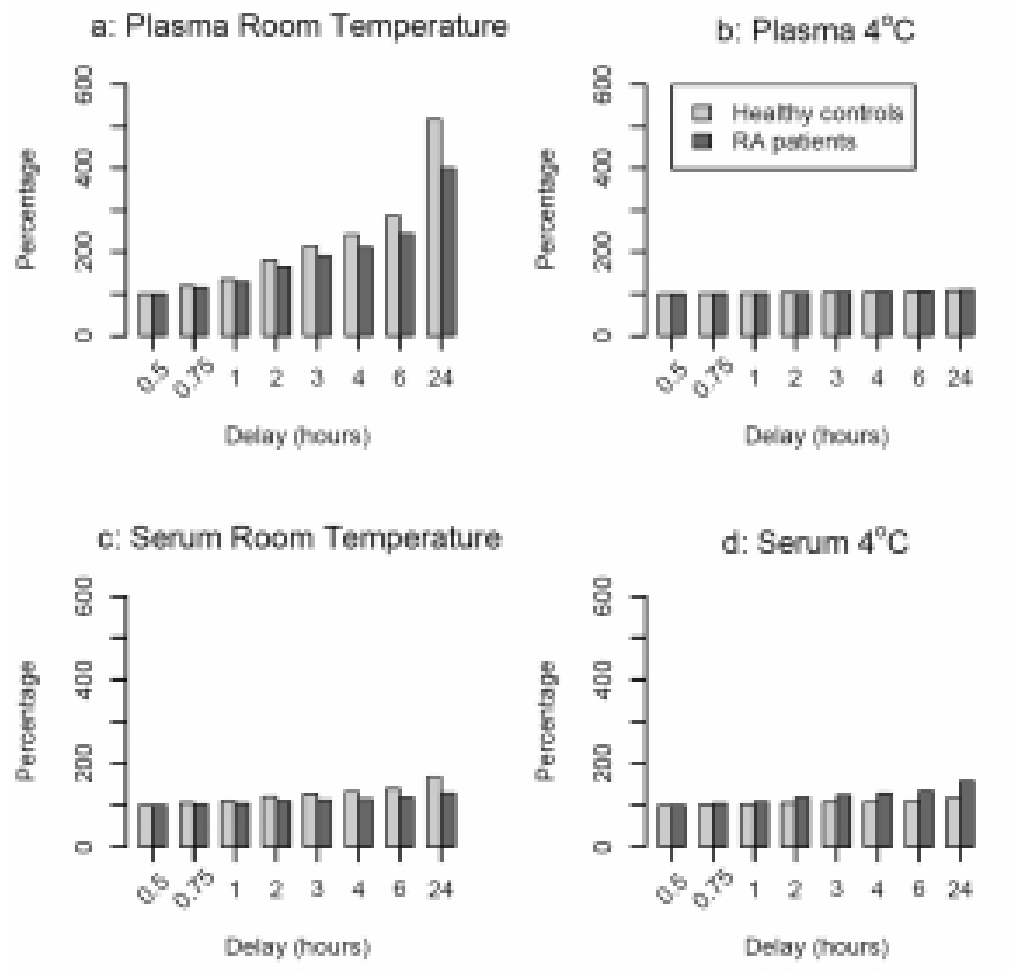

Fig. 1. Impact of delay of centrifugation. The percent changes in plasma VEGF (from 0.5 hours) at room temperature (a) and $4 \mathrm{C}$ (b) and in serum VEGF at room temperature (c) and $4^{\circ} \mathrm{C}(\mathrm{d})$ in healthy persons (light columns) and patients with rheumatoid arthritis (dark columns) are shown.

\subsubsection{Intra- and interassay $C V$}

The intra-assay CVs for plasma samples with VEGF levels around the detection limit was $17.4 \%$ (mean plasma VEGF $14.1 \mathrm{pg} / \mathrm{ml}, n=34$ ). In plasma control samples with high VEGF concentration it was $4.0 \%$ (169 $\mathrm{pg} / \mathrm{ml}, n=35)$. The intra-assay CVs for two serum samples with low or high VEGF concentrations were $11.3 \%$ (223 pg/ml, $n=36)$ and $4.1 \%(764 \mathrm{pg} / \mathrm{ml}$, $n=34)$, respectively. Inter-assay CV over a period of 21 months using 5 different ELISA batches was $9.1 \%$ (mean VEGF $216 \mathrm{pg} / \mathrm{ml}, n=378$ ), 9.7\% (374 pg/ml, $n=66)$ and $12.8 \%$ (487 pg/ml, $n=416)$.

\subsubsection{Recovery}

The observed final concentration as a proportion of the expected final concentration was 1.03 for serum VEGF and 1.02 for EDTA plasma VEGF, in accordance with the manufacturer's specifications.

\subsubsection{Delay and temperature before centrifugation}

Figure 1 shows the mean percent changes in VEGF after delays of processing from 0.5 to 24 hours com- pared to baseline values. Plasma VEGF stored at $4{ }^{\circ} \mathrm{C}$ from healthy persons and patients did not increase significantly over time for up to 24 hours $(p=0.79$ and $p=0.50$, respectively). For the same samples stored at room temperature, plasma VEGF was significantly associated to processing time $(p<0.0001)$ for both healthy persons and patients, and had increased by $34 \%$ (after 1 hour) and $80 \%$ (after 2 hours) in healthy persons; in RA patients it was $28 \%$ and $64 \%$, respectively. Serum VEGF was significantly associated to processing time at both temperatures. At $4{ }^{\circ} \mathrm{C}$, the increase was 3\% (after 1 hour) and 6\% (after 2 hours) in healthy persons $(p=0.04) ; 9 \%$ and $18 \%$ in RA patients $(p=$ 0.0006 ); at room temperature it was $10 \%$ and $21 \%$ in healthy persons $(p<0.0001)$, and $5 \%$ and $10 \%$ in RA patients $(p=0.0004)$.

\subsubsection{Centrifugation and strata}

In healthy persons significantly lower plasma VEGF was found for samples prepared at $3000 \mathrm{~g}$ (geometric mean $31 \mathrm{pg} / \mathrm{ml}$ (non-detectable (ND)-105)) compared to $2000 \mathrm{~g}$ (40 pg/ml (ND-261) (22\% lower), $p=0.007$. 
Table 1

VEGF concentrations $(\mathrm{pg} / \mathrm{ml})$ in different strata of EDTA plasma and serum according to different centrifugation procedures in patients with rheumatoid arthritis

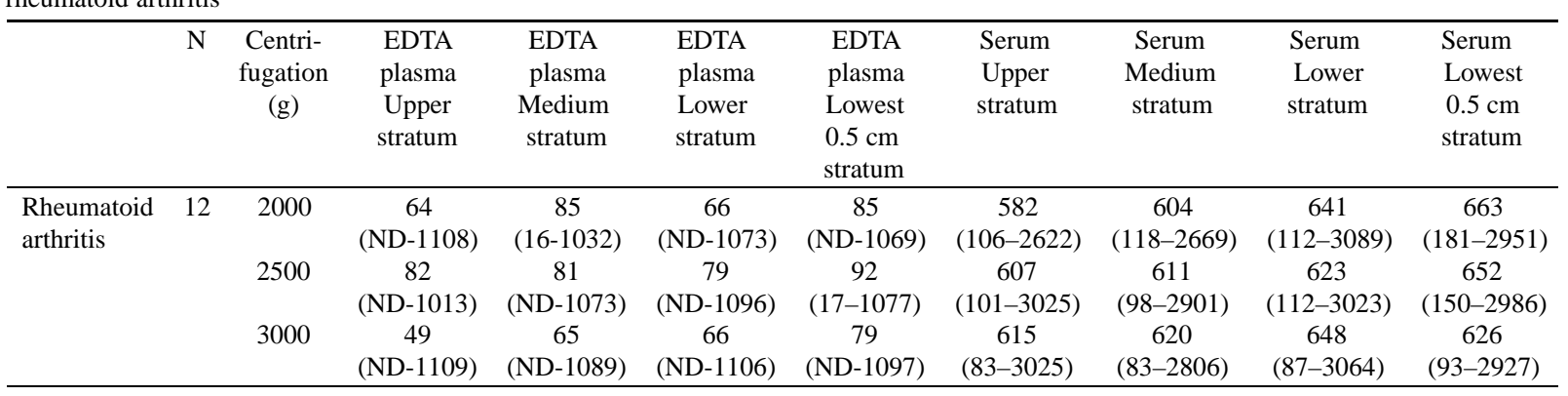

VEGF: Vascular endothelial growth factor. Values are geometrical means (range). ND = not detectable $(<16 \mathrm{pg} / \mathrm{ml})$.

Table 2

VEGF concentrations $(\mathrm{pg} / \mathrm{ml})$ in different types of plasma and serum in healthy persons and patients with rheumatoid arthritis

\begin{tabular}{lllcccc}
\hline & $\mathrm{N}$ & EDTA plasma & EDTA plasma on ice & Heparin plasma & Citrate plasma & Serum \\
\hline Healthy persons & 10 & ND (ND-69)* & ND (ND-85) & 32 (ND-64) & $17(17-54)$ & $313(96-494)$ \\
Rheumatoid arthritis par & 10 & 40 (ND-144)* & 16 (ND-78) & 34 (ND-157) & $18(17-47)$ & $492(113-924)$ \\
\hline
\end{tabular}

VEGF: Vascular endothelial growth factor. Values are medians (range). Kruskal-Wallis rank sum test for differences between groups.

*** $: p<0.001$ for difference between serum and each of the plasma groups.

${ }^{*} p=0.01$ for difference between healthy persons and RA patients.

Centrifugation at $1200 \mathrm{~g}$ resulted in significantly higher plasma VEGF (56-67 pg/ml) (61-91\% higher) than at $2200 \mathrm{~g}(35-48 \mathrm{pg} / \mathrm{ml}), p=0.03$ with intermediate values at $1700 \mathrm{~g}(43-51 \mathrm{pg} / \mathrm{ml})$. Plasma VEGF was significantly higher in the supernatant closest to the buffy coat (48-67 pg/ml) compared to the top stratum (43-57 pg/ml), $p=0.01$. In RA patients, centrifugation at $2500 \mathrm{~g}$ resulted in $31 \%$ higher concentration of plasma VEGF than at $3000 \mathrm{~g}(p=0.01)$, with highest concentration in the lower and the lowest stratum $(p<$ 0.0001 ), Table 1. No significant interactions between centrifugation speed and plasma stratum could be detected. Serum VEGF was not significantly related to centrifugal speed or strata.

\subsubsection{Different types of blood samples and temperature}

Table 2 shows the VEGF concentrations in different types of blood samples in healthy persons and RA patients. In both groups serum VEGF was several times higher than in the different types of plasma. Heparin plasma and EDTA plasma (room temperature) had similar VEGF concentration, whereas significantly lower levels were found in citrate plasma. Collection of EDTA plasma on ice resulted in lower levels of VEGF than collection at room temperature.

\subsubsection{Buffy coat contamination}

Plasma VEGF was 33\% higher in the samples that contained the supernatant closest to the buffy coat compared to the samples without $(p=0.01)$.

\subsubsection{Repeated thawing}

Plasma VEGF was significantly higher in a sample that had only been thawed once compared to thawing 2 to 10 times (mean difference $20 \%, p=0.005$, GLM repeated measures adjusted for normal/patient). No difference could be demonstrated for plasma samples thawed from 2 to 9 times, whereas a significantly lower level was found for the $10^{\text {th }}$ thaw (mean difference $8 \%), p=0.003)$. Serum VEGF levels were not significantly different if thawed from 1 to 9 times whereas the concentration was lower after 10 thaws (mean difference $18 \%$ ), $p=0.003$ ).

\subsubsection{Storage}

In the initial analyses, the plasma VEGF was median $88 \mathrm{pg} / \mathrm{ml}$ (range ND-537), and in the reanalyses after 2 years it was $85 \mathrm{pg} / \mathrm{ml}$ (ND-601). The median difference was $1 \mathrm{pg} / \mathrm{ml}(-85-24), p=0.27$.

\subsection{Biological variability}

\subsubsection{Reference interval (normal range)}

Figure 2 illustrates the individual VEGF concentrations in plasma (a) and serum (b) in 306 healthy persons according to age and gender. The median plasma VEGF was 45 pg/ml (range: ND-351; 5-95\% interval: ND-137). Serum VEGF was 328 pg/ml (range: 531791; 5-95\% interval: 118-990). The correlation between VEGF concentrations in plasma and serum was 
(a)

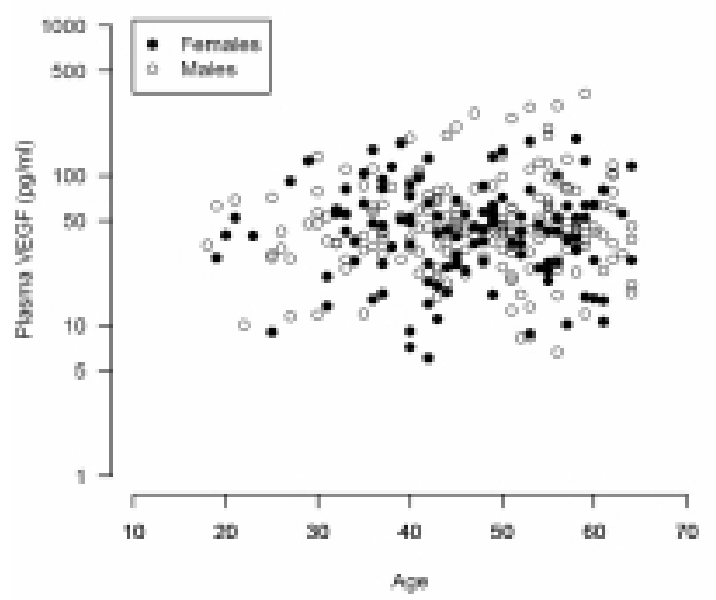

(b)

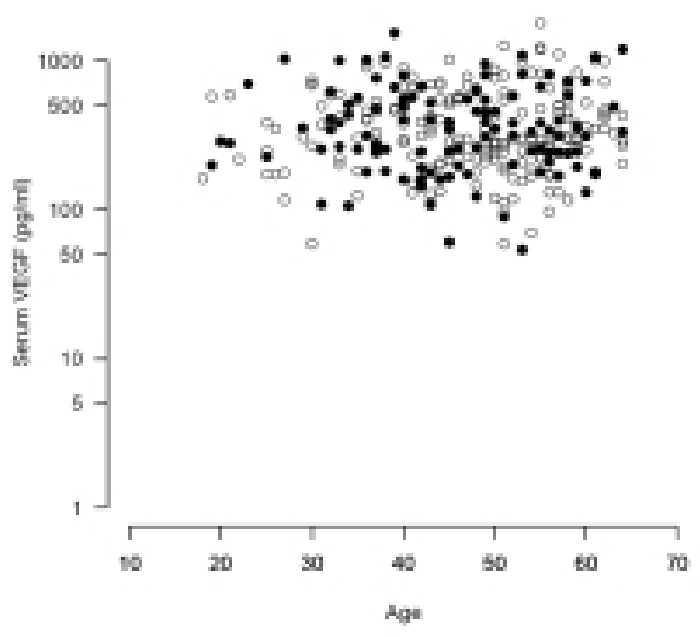

Fig. 2. Individual VEGF concentrations in EDTA plasma (a) and serum (b) in 306 healthy persons according to age and sex. The $\mathrm{y}$-axis is a $\log$ scale.

$r=0.67, p<0.0001$. There was no difference between men and women (plasma: $p=0.25$; serum: $p=$ 0.92 ) and no correlation to age (plasma: $r=0.001$, $p=0.99$; serum: $r=-0.01, p=0.88$ ).

\subsubsection{Diurnal variability}

Figure 3 shows the diurnal changes in plasma VEGF in healthy persons (a), in patients with early, untreated RA (b), and in patients with established, erosive RA (c). Comparing plasma VEGF in the three groups did not demonstrate statistical significance (healthy versus early RA, $p=0.26$; healthy versus established RA,
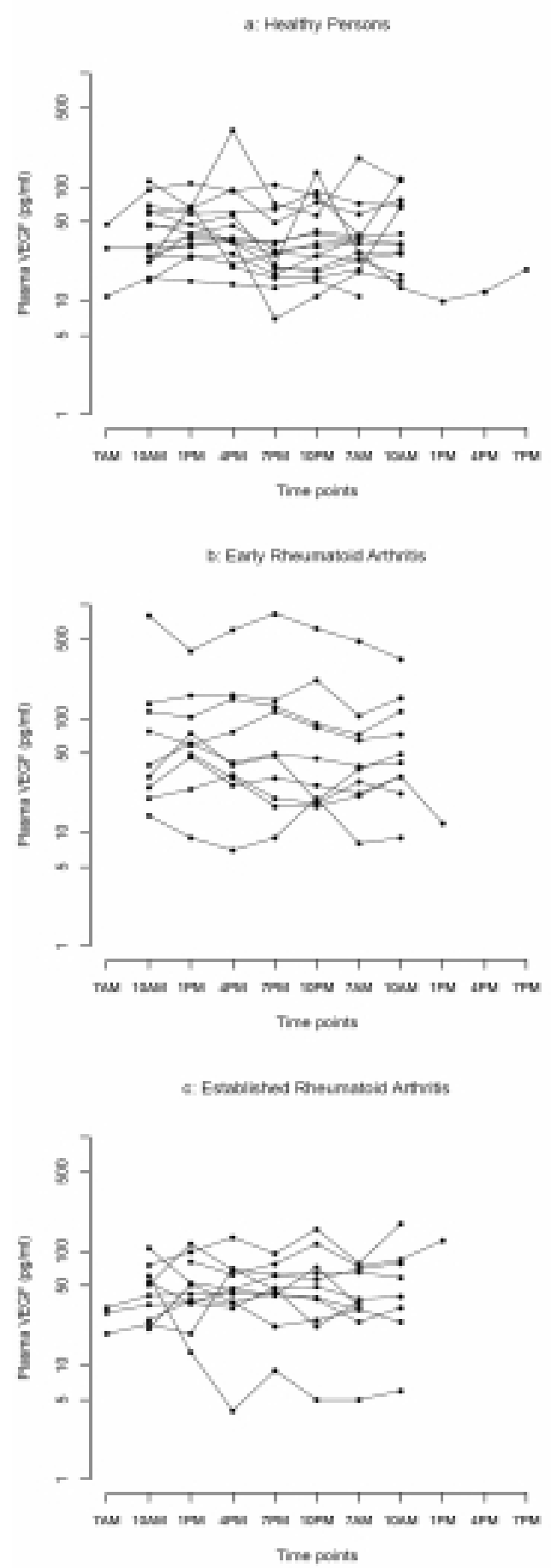

Fig. 3. The diurnal changes in plasma VEGF in healthy persons (a), and in patients with early rheumatoid arthritis (b) and established, erosive rheumatoid arthritis (c). The y-axis is a log scale.

$p=0.82$; and early RA versus established RA, $p=$ 0.42). A linear model adjusting for group showed that 
plasma VEGF at 7 AM was significantly lower than the samples at $1 \mathrm{PM}$ (27\% higher, $p=0.007)$ and $4 \mathrm{PM}$ (18\% higher, $p=0.006$ ).

\subsubsection{Variability during 3 weeks}

The median day to day $\mathrm{CV}$ of plasma VEGF for each subject (all samples taken at $8 \mathrm{PM}$ ) was $48 \%$ (23$126 \%)$ for the $1^{\text {st }}$ round, $56 \%(22-122 \%)$ six months later $\left(2^{\text {nd }}\right)$, and 39\% (11-149\%) 12 months later $\left(3^{\text {rd }}\right)$. Comparing the sample taken at $2 \mathrm{PM}$ to the sample taken at $8 \mathrm{PM}$ on the same day for each subject could not demonstrate a systematic difference: $1^{\text {st }}$ round: $p=0.09 ; 2^{\text {nd }}$ round: $p=0.84 ; 3^{\text {rd }}$ round: $p=0.13$.

\subsubsection{Long-term variability}

The plasma VEGF levels were significantly different between the three rounds $(p<0.0001)$ : The median plasma VEGF for each person initially, 6 months later and 1 year later were $21 \mathrm{pg} / \mathrm{ml}$ (16-131), $16 \mathrm{pg} / \mathrm{ml}$ (16-43) and $51 \mathrm{pg} / \mathrm{ml}(21-309)$, respectively.

\subsubsection{Exercise}

Figure 4 shows the individual changes in plasma VEGF before and after 25 minutes of bicycling in healthy persons (a), in patients with early RA (b) and in patients with established, erosive RA (c). Plasma VEGF was increased immediately after bicycling in the healthy persons (16\% higher, $p=0.04)$ and in the patients with erosive RA $(64 \%, p=0.0002)$, but not in early RA.

\section{Discussion}

Circulating VEGF has been suggested as a possible biomarker for the monitoring of disease activity, treatment response and erosive progression in patients with RA. Thus, serum VEGF is elevated in patients with active RA compared to healthy subjects, and it has been reported to correlate to serum CRP, the number of swollen joints and disease activity score, and to decrease in RA patients, who respond to diseasemodifying treatment and tumour-necrosis factor alpha inhibitors $[2,9,16,18,24]$. One study reported that serum VEGF level in patients with early RA was correlated with the progression of joint destruction after one year [2].

The lack of methodological studies of circulating VEGF in RA patients prompted us to perform the present studies. The results show that the contribution of pre-analytical factors such as delayed process-
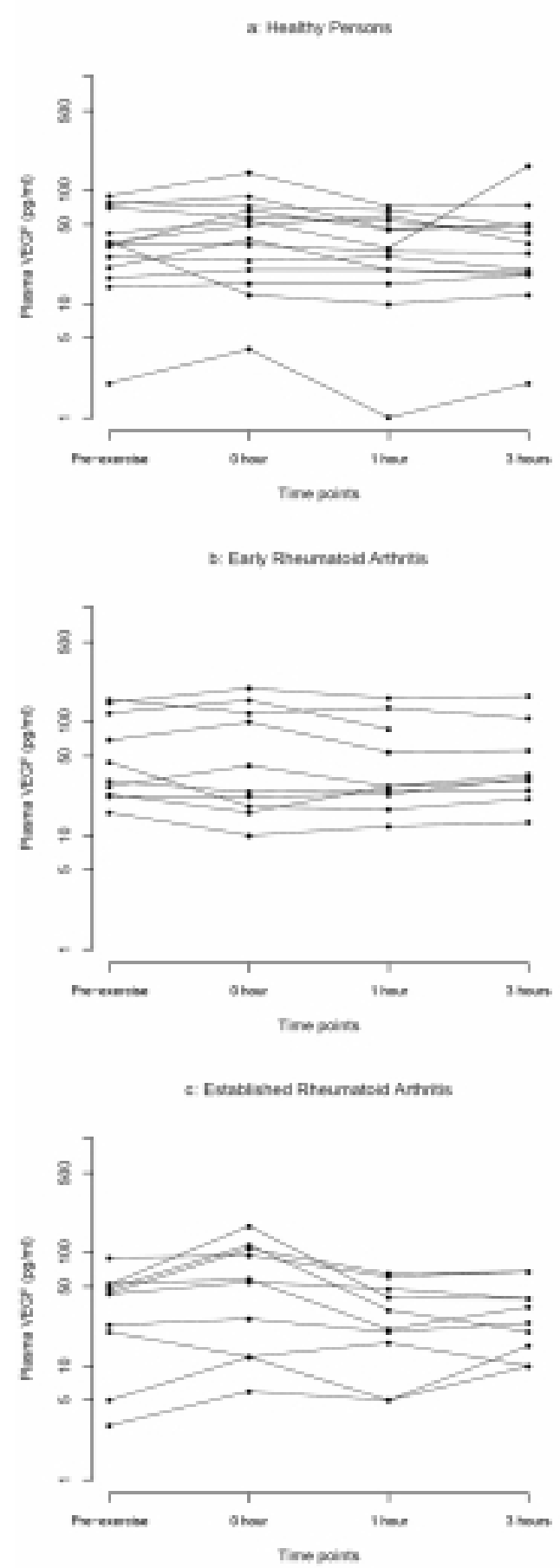

Fig. 4. The changes after 12 min of bicycling in plasma VEGF in healthy persons (a), and in patients with early rheumatoid arthritis (b) and established, erosive rheumatoid arthritis (c). The individual fluctuations are shown. The y-axis is a log scale.

ing time, low centrifugal force, contamination of plasma with cellular content and high temperature before 
centrifugation, all leading to increased in vitro concentrations of VEGF, should be taken into account in the interpretation of data in RA studies. It confirms and expands previous findings in healthy persons [10]. VEGF in serum was 7 to 10 fold higher than in plasma, which put a question mark against the suitability of serum VEGF as a biomarker.

Low centrifugal force and sampling from the supernatant close to the buffy coat resulted in higher plasma VEGF. This is probably caused by a higher cellular content of the sample, and is in accordance with a previous study [10]. The variation in plasma VEGF in the different strata of the supernatant was large enough to confound the measurement considerably and should be taken into account when VEGF is analysed in clinical studies. The findings stress the significance of standardized sampling procedures (e.g. leaving $5 \mathrm{~mm}$ of the supernatant above the buffy coat untouched, aliquot the supernatant in one pipetting procedure, apply uniform centrifugal force with a least $1700 \mathrm{~g}$ across laboratories).

It has not previously been discussed whether serum or plasma should be recommended for analysis of VEGF as a potential biomarker of angiogenesis in studies of patients with rheumatoid arthritis. In vivo, the synovium is perfused by whole blood, thus being exposed to growth factors present in the plasma [10]. In contrast, growth factors in serum reflect concentrations after coagulation, which rarely occurs in vivo. Thus, during blood clotting, a time-dependant efflux of VEGF from platelets and neutrophils take place, leading to increased serum concentrations compared to plasma [3, $8,26,27]$. In accordance with this, we found that serum VEGF was several fold higher than the corresponding plasma samples both in patients with RA (approximately 10 times higher) and in healthy persons ( 7 times).

VEGF levels in EDTA and heparin plasma were similar. The lowest VEGF levels were seen in citrated plasma, indicating that prevention of the release of VEGF from platelets was most efficient there. The difference was almost eliminated when the collection of EDTA plasma was done on ice. In contrast, others have found that cold temperatures tend to elevate plasma VEGF [10].

In agreement with others, we found that serum VEGF was stable for up to 9 freeze-thaw cycles $[23,26]$, and that plasma VEGF was equally stable for 2 to 9 cycles. This should be taken into account, when samples are frozen and thawed several times before analysis. Storage of plasma for 2 years at $-80^{\circ} \mathrm{C}$ did not result in any systematic change in VEGF levels.
We established a reference interval of corresponding serum and plasma VEGF concentrations in 306 blood donors, and found that VEGF levels were unrelated to gender and age. Our reference interval for plasma VEGF is similar to that found in a recent cohort study [4]. In contrast, our reference interval for serum VEGF is higher than what was found in 136 healthy persons in a colo-rectal cancer study [14] (median 328 vs. $174 \mathrm{pg} / \mathrm{ml}$ ). It is not stated how the healthy persons were recruited into this study. In another colo-rectal cancer study [7], plasma and serum VEGF were 9 pg/ml and $152 \mathrm{pg} / \mathrm{ml}$, respectively in 116 persons with benign colon disease. In this study, citrated plasma was used, which may in part explain the lower plasma values. In the cohort study [4] there was a trend for higher plasma VEGF in women than in men, which is in contrast to our findings. The assay variation was, as expected, high at low VEGF concentrations due to the shape of the standard curve. This should be taken into account in plasma samples in normal individuals, who have low VEGF concentrations.

In both RA and in healthy persons no systematic diurnal variation could be documented, except that 7 AM samples were significantly lower than afternoon samples. This has the practical consequence that the sampling may take place at any time during the day after 7 AM.

Changes in plasma VEGF during one month were measured at 5 time points and were repeated again after 6 and 12 months. During the first and second period of sampling, some individuals showed large fluctuations over time. The fluctuations were not systematic and gave suspicion to pre-analytic variability. The sampling procedure was therefore altered during the third period of sampling to avoid the VEGF concentration gradient in plasma: The whole supernatant was aliquoted in one pipette, leaving the $5 \mathrm{~mm}$ stratum closest to the buffy coat untouched. Afterwards the supernatant was divided into four tubes. This reduced, but did not eliminate the longterm variability, which indicates that also biological variability contributed to the fluctuations.

We found increased levels of plasma VEGF in healthy persons and in patients with established RA right after bicycling. This did not apply to patients with early RA, probably because their widespread joint pain resulted in less vigorous exercise. The impact of physical activity may have contributed to the longterm variability, in which the study conditions were less standardised, allowing some light exercise prior to blood sampling like bi-cycling to work and brisk walking while at work. 
In conclusion, pre-analytical factors should be accounted for and reported in studies regarding circulating VEGF. We established a reference interval of serum and plasma VEGF in healthy persons. Measurement of VEGF in serum lead to several fold higher concentrations than in plasma, probably reflecting that serum VEGF is a combination of both the VEGF from platelet release on coagulation and the circulating VEGF. Significant short- and long-term biologic variability included diurnal variation and impact of physical activity, which should be standardized in future clinical studies.

\section{Acknowledgments}

The expert technical assistance of Teresa Rozenfeld, Birgitte Sander Nielsen and Susanne Reimer at Copenhagen University Hospital at Hvidovre are gratefully acknowledged. The staff of Department of Clinical Immunology, Nykøbing Falster County Hospital is thanked for collection of blood samples from healthy volunteer blood donors.

\section{Sources of support}

The study was supported by grants from the Danish Rheumatism Association, The Copenhagen Hospital Corporation, Grosserer Valdemar Foersom og hustru Thyra Foersom, født Otto's fond, Henny og Helge Holgersens Mindelegat, Overlæge Johan Boserup og Lise Boserups legat, The Institute of Clinical Research at University of Southern Denmark, The Kornerup Fund, The Aase and Ejnar Danielsen Fund, The Aage and Johanne Louis-Hansen Fund and the Danish Cancer Society. The funding organizations had no role in the design of the study, data collection, analysis and interpretation, preparation of the manuscript, and had no rights to approve, delay or disapprove publication of the work.

\section{References}

[1] M.G. Achen and S.A. Stacker, The vascular endothelial growth factor family; proteins which guide the development of the vasculature, Int J Exp Pathol 79 (1998), 255-265.

[2] S. Ballara, P.C. Taylor, P. Reusch, D. Marme, M. Feldmann, R.N. Maini and E.M. Paleolog, Raised serum vascular endothelial growth factor levels are associated with destructive change in inflammatory arthritis 1, Arthritis Rheum 44 (2001), 2055-2064.
[3] R.E. Banks, M.A. Forbes, S.E. Kinsey, A. Stanley, E. Ingham, C. Walters and P.J. Selby, Release of the angiogenic cytokine vascular endothelial growth factor (VEGF) from platelets: significance for VEGF measurements and cancer biology, $\mathrm{Br} \mathrm{J}$ Cancer 77 (1998), 956-964.

[4] H. Berrahmoune, J.V. Lamont, B. Herbeth, P.S. FitzGerald and S. Visvikis-Siest, Biological determinants of and reference values for plasma interleukin-8, monocyte chemoattractant protein-1, epidermal growth factor, and vascular endothelial growth factor: Results from the STANISLAS cohort, Clin Chem 52 (2006), 504-510.

[5] L.F. Brown, M. Detmar, K. Claffey, J.A. Nagy, D. Feng, A.M. Dvorak and H.F. Dvorak, Vascular permeability factor/vascular endothelial growth factor: a multifunctional angiogenic cytokine, EXS 79 (1997), 233-269.

[6] R.A. Fava, N.J. Olsen, G. Spencer-Green, K.T. Yeo, T.K. Yeo, B. Berse, R.W. Jackman, D.R. Senger, H.F. Dvorak and L.F. Brown, Vascular permeability factor/endothelial growth factor (VPF/VEGF): accumulation and expression in human synovial fluids and rheumatoid synovial tissue, J Exp Med 180 (1994), 341-346.

[7] M.L. George, S.A. Eccles, M.G. Tutton, A.M. Abulafi and R.I. Swift, Correlation of plasma and serum vascular endothelial growth factor levels with platelet count in colorectal cancer: clinical evidence of platelet scavenging? Clin Cancer Res 6 (2000), 3147-3152.

[8] E. Gunsilius, A. Petzer, G. Stockhammer, W. Nussbaumer, P. Schumacher, J. Clausen and G. Gastl, Thrombocytes are the major source for soluble vascular endothelial growth factor in peripheral blood, Oncology 58 (2000), 169-174.

[9] M. Harada, K. Mitsuyama, H. Yoshida, S. Sakisaka, E. Taniguchi, T. Kawaguchi, M. Ariyoshi, T. Saiki, M. Sakamoto, K. Nagata, M. Sata, K. Matsuo and K. Tanikawa, Vascular endothelial growth factor in patients with rheumatoid arthritis, Scand J Rheumatol 27 (1998), 377-380.

[10] E. Hormbrey, P. Gillespie, K. Turner, C. Han, A. Roberts, D. McGrouther and A.L. Harris, A critical review of vascular endothelial growth factor (VEGF) analysis in peripheral blood: is the current literature meaningful? Clin Exp Metastasis 19 (2002), 651-663.

[11] J.R. Jackson, J.A. Minton, M.L. Ho, N. Wei and J.D. Winkler, Expression of vascular endothelial growth factor in synovial fibroblasts is induced by hypoxia and interleukin 1beta, J Rheumatol 24 (1997), 1253-1259.

[12] A.E. Koch, Review: angiogenesis: implications for rheumatoid arthritis, Arthritis Rheum 41 (1998), 951-962.

[13] A.E. Koch, L.A. Harlow, G.K. Haines, E.P. Amento, E.N. Unemori, W.L. Wong, R.M. Pope and N. Ferrara, Vascular endothelial growth factor. A cytokine modulating endothelial function in rheumatoid arthritis, J Immunol 152 (1994), 41494156.

[14] H. Kumar, K. Heer, P.W. Lee, G.S. Duthie, A.W. MacDonald, J. Greenman, M.J. Kerin and J.R. Monson. Preoperative serum vascular endothelial growth factor can predict stage in colorectal cancer, Clin Cancer Res 4 (1998), 1279-1285.

[15] A. Kuryliszyn-Moskal, P.A. Klimiuk, S. Sierakowski and M. Ciolkiewicz, A study on vascular endothelial growth factor and endothelin-1 in patients with extra-articular involvement of rheumatoid arthritis, Clin Rheumatol (2005), 1-6.

[16] S.S. Lee, Y.S. Joo, W.U. Kim, D.J. Min, J.K. Min, S.H. Park, C.S. Cho and H.Y. Kim, Vascular endothelial growth factor levels in the serum and synovial fluid of patients with rheumatoid arthritis, Clin Exp Rheumatol 19 (2001), 321-324. 
[17] I. Macias, S. Garcia-Perez, M. Ruiz-Tudela, F. Medina, N. Chozas and J.A. Giron-Gonzalez, Modification of pro- and antiinflammatory cytokines and vascular-related molecules by tumor necrosis factor-a blockade in patients with rheumatoid arthritis, J Rheumatol 32 (2005), 2102-2108.

[18] M. Nagashima, S. Yoshino, T. Ishiwata and G. Asano, Role of vascular endothelial growth factor in angiogenesis of rheumatoid arthritis, J Rheumatol 22 (1995), 1624-1630.

[19] E.M. Paleolog, Angiogenesis: a critical process in the pathogenesis of RA-a role for VEGF? 2, Br J Rheumatol 35 (1996), 917-919.

[20] E.M. Paleolog, Angiogenesis in rheumatoid arthritis, Arthritis Res 4(Suppl 3) (2002), S81-S90.

[21] E.M. Paleolog and J.M. Miotla, Angiogenesis in arthritis: role in disease pathogenesis and as a potential therapeutic target, Angiogenesis 2 (1998), 295-307.

[22] E.M. Paleolog, S. Young, A.C. Stark, R.V. McCloskey, M. Feldmann and R.N. Maini, Modulation of angiogenic vascular endothelial growth factor by tumor necrosis factor alpha and interleukin-1 in rheumatoid arthritis, Arthritis Rheum 41 (1998), 1258-1265.
[23] P. Salven, L. Teerenhovi and H. Joensuu, A high pretreatment serum vascular endothelial growth factor concentration is associated with poor outcome in non-Hodgkin's lymphoma, Blood 90 (1997), 3167-3172.

[24] H. Sone, M. Sakauchi, A. Takahashi, H. Suzuki, N. Inoue, K. Iida, H. Shimano, H. Toyoshima, Y. Kawakami, Y. Okuda, K. Matsuo and N. Yamada, Elevated levels of vascular endothelial growth factor in the sera of patients with rheumatoid arthritis correlation with disease activity, Life Sci 69 (2001), 18611869.

[25] D.A. Walsh, Angiogenesis and arthritis, Rheumatology (Oxford) 38 (1999), 103-112.

[26] N.J. Webb, M.J. Bottomley, C.J. Watson and P.E. Brenchley, Vascular endothelial growth factor (VEGF) is released from platelets during blood clotting: implications for measurement of circulating VEGF levels in clinical disease, Clin Sci (Lond) 94 (1998), 395-404.

[27] K. Werther, I.J. Christensen and H.J. Nielsen, Determination of vascular endothelial growth factor (VEGF) in circulating blood: significance of VEGF in various leucocytes and platelets, Scand J Clin Lab Invest 62 (2002), 343-350. 


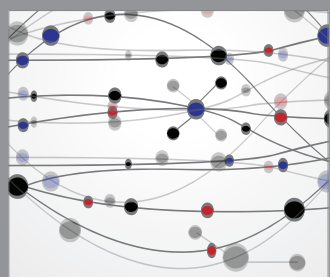

The Scientific World Journal
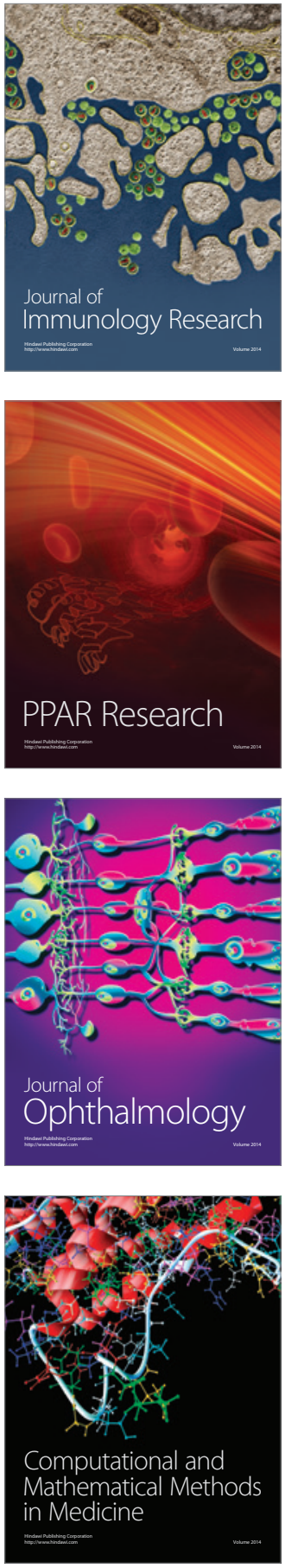

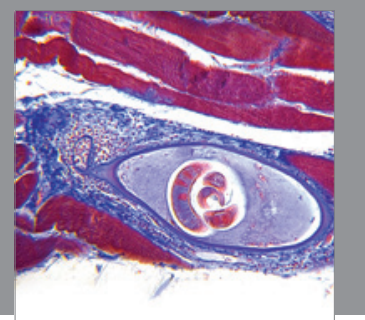

Gastroenterology

Research and Practice
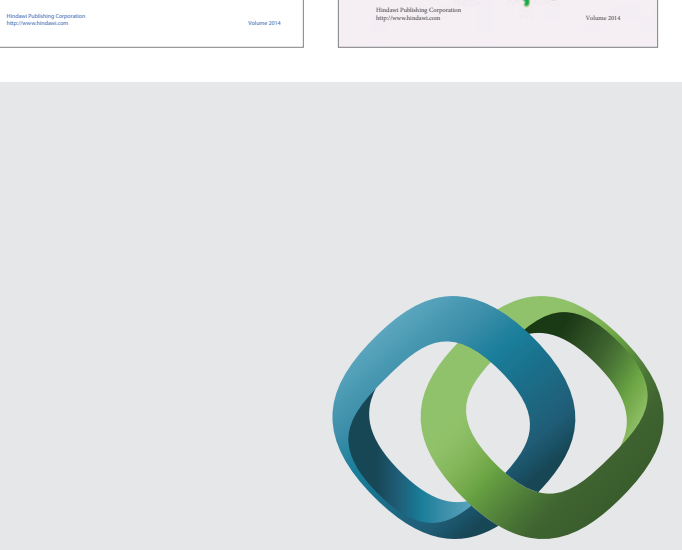

\section{Hindawi}

Submit your manuscripts at

http://www.hindawi.com
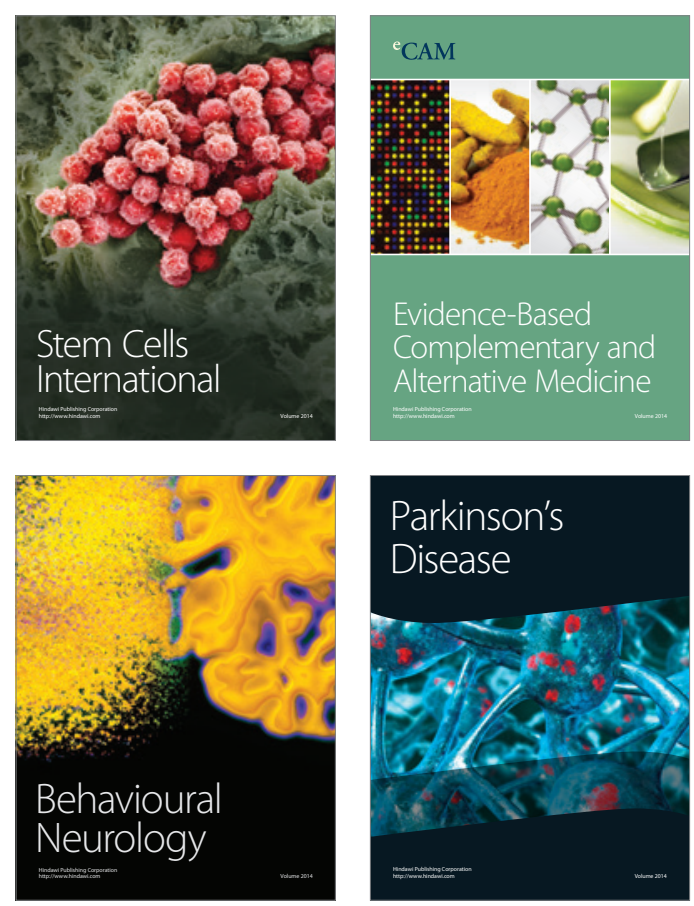

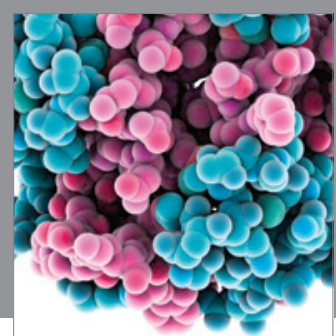

Journal of
Diabetes Research

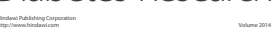

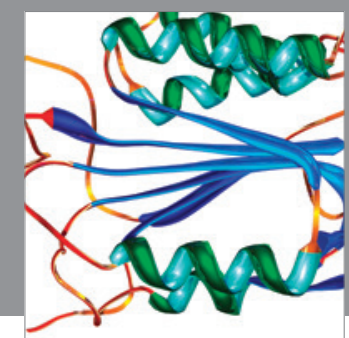

Disease Markers
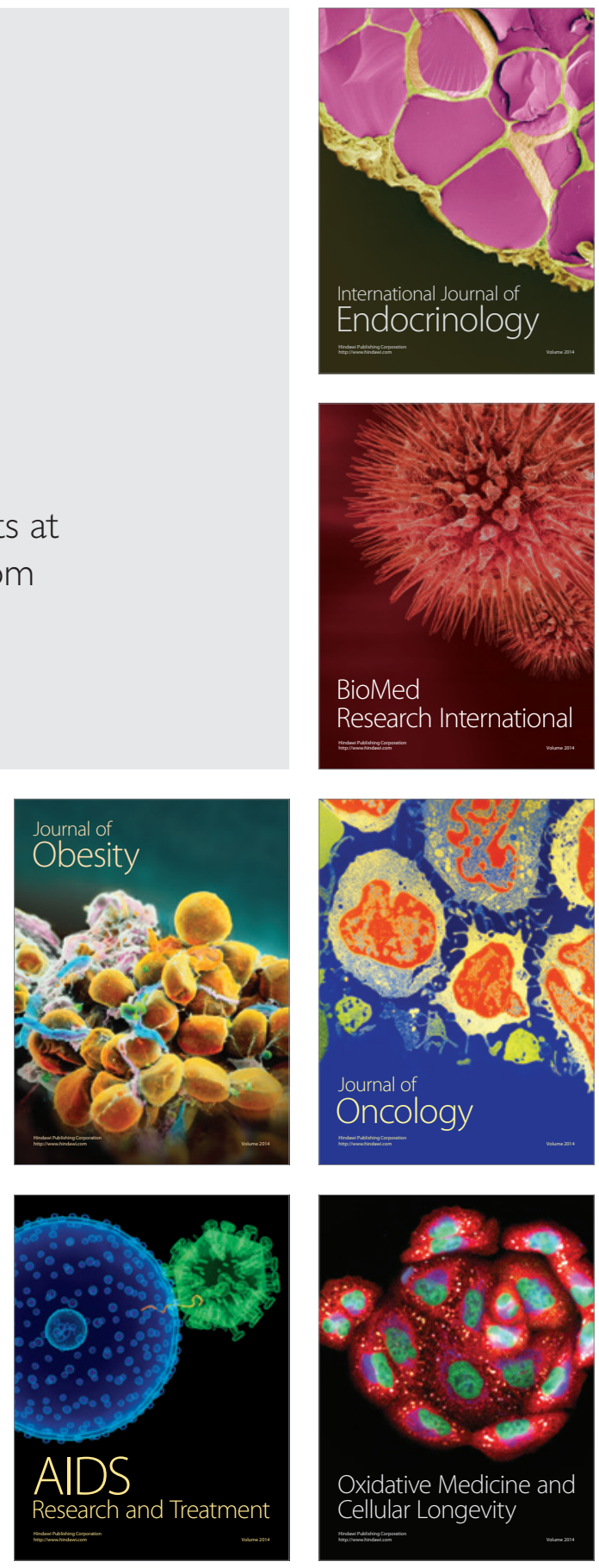